\title{
Detecting Abnormalities in MRI Images Using the Difference Method
}

\author{
Kwee Hiong Lee \\ Neural Systems Engineering Laboratory, Department of Electrical Engineering \\ Imperial College of Science, Technology and Medicine \\ Exhibition Road, London SW7 2BT
}

\begin{abstract}
With advances in MRI techniques and equipment, it is possible to obtain images of good contrast or high resolution. Taking one step further is to develop a system which processes these images and detects abnormalities within them with reasonable accuracy. This paper describes such an application using neural networks on MRI images of the human head.

As the two halves of the human brain are approximately mirror-image of the other [1], this symmetry property can be used. Hence, instead of operating directly on the image, the Difference Operation is applied on symmetrical areas of the image. Before this, a pre-processing step is needed to position the head (within the image) so that it occupies the top central position in the image. The Difference Operation involves evaluating the absolute difference in intensity between pixels taken from two regions that occupy symmetrical positions in the image. Pairs of pixels are taken with the first from one region and the other from the symmetrical position in the second region. The resulting difference map is operated on by the neural network.

The neural network is a discriminator-based system similar to WISARD [2]. Instead of using Random Access Memory (RAM) as nodes, Multi-valued Probabilistic Logic Nodes (MPLNs) [3] are used. The inputs to the nodes are taken randomly from the input image. As the input to the nodes are binary while the pixel intensities from the image are in 256 gray levels, an intensity coding module is needed. Each input is assigned a buffer with a certain number of memory locations in it. The specified intensity range is divided uniformly among these memory locations which are initially set to " 0.5 ". With training, the contents are increased to " 1 " or decreased to " 0 ". This will form a define mapping from a gray scale input to a binary output for each input.

Three main parameters were varied in the simulations. For intensity coding, the number of intensity ranges and the number of gray levels in each range were varied. The product of these two parameters was used as the upper limit of the allowed intensity range. Thus, any pixel intensity above this limit would be set to it. The number of training iterations was also varied from 1 times to 16 times. From the results, it is clear that proposed system can be used to detect abnormalities. Using a small number of intensity ranges and a medium number of gray levels in each range gives the best detection. The detection rate increases with training but decreases after reaching an optimum number of training iterations.
\end{abstract}

\section{References}

[1] Springer, S.P. and Deutsch, G., "Left Brain, Right Brain", Freeman, San Francisco, 1981.

[2] Aleksander,I.;Thomas,W.V. and Bowden,P.A., "WISARD, a radical step forward in image recognition", Sensor Review,vol.4,no 3,1984.

[3] Aleksander,I., "The Logic of Connectionist Systems", in Aleksander,I., eds., "Neural Computing Architectures", Boston, MIT press, 1989. 